\title{
HISTOIRE DES IDÉES, SOCIOLOGIE DES CROYANCES ET PROCESSUS ARGUMENTATIFS SCEPTICISME ET MODERNITÉ D'APRÈS RICHARD H. POPKIN
}

\begin{abstract}
Alban Bouvier
Résumé: On se propose, dans cet article, de montrer l'intérêt de l'ouvrage classique de Richard H. Popkin du point de vue d'une analyse sociologique et anthropologique des processus collectifs de doute et d'adhésion. Un tel examen implique toutefois, au niveau de la méthode, d'analyser les différences fines existant entre histoire des idées, sociologie de la connaissance, ethnologie des croyances et épistémologie. L'examen mérite d'être mené aussi bien pour la compréhension du développement du scepticisme au Xvil siècle que du déferlement de la vague relativiste contemporaine, qu'il faut assurément distinguer, en suivant l'inspiration de Popkin, d'une forme de scepticisme modéré proche du rationalisme critique.
\end{abstract}

MoTS-CLÉs : argumentation, croyances collectives, Descartes, effets contre-intentionnels, Laudan, Popper, relativisme contemporain, rhétorique, scepticisme classique, sociologie de la connaissance.

ABSTRACT: This paper claims to show the relevance of the classical book by Richard H. Popkin from the point of view of a sociological and anthropological analysis of the collective processes of doubt and assent. This examination implies, however, that we analyse the fine differences between the history of ideas, the sociology of knowledge, the ethnology of beliefs and epistemology. The examination deserves to be carried out as well for the understanding of the increase of scepticism in the 17th century as for the spread of the wave of contemporary relativism. The latter certainly has to be distinguished, as Popkin suggests, from a form of moderate scepticism close to critical rationalism.

KEYWORDS : argumentation, collective beliefs, Descartes, unintended effects, Laudan, Popper, contemporary relativism, rhetoric, classical scepticism, sociology of knowledge.

Revue de synthèse : $4^{\text {e }}$ S. $\mathrm{n}^{\text {os }}$ 2-3, avr.-sept. 1998, p. 307-322. 
ZusAMMENFASSUNG: In diesem Aufsatz wird beabsichtigt, die Bedeutung von Richard H. Popkins klassischem Werk, vom Standpunkt einer soziologischen und anthropologischen Analyse der kollektiven Zweifel- und Zustimmungsprozesse zu beweisen. Eine solche Erforschung bringt jedoch mit sich, was die Methode anbelangt, die feinen Unterschiede zwischen Gedankengeschichte, Wissensoziologie, Glaubensanthropologie und Wissenschaftslehre zu erforschen. Es lohnt sich die Untersuchung um des Verständnisses sowohl der Entwicklung des Skeptizismus im 17. Jahrhundert als auch der lawinenartigen Vermehrung des gegenwärtigen Relativismus willens zu unternehmen - der natürlich, Popkins Eingebung folgend, von einem gemässigten, dem kritischen Rationalismus nahestehenden Skeptizismus unterschieden werden soll.

STICHWÖRTER : Argumentation, Kollectivglaube, Descartes, Laudan, Popper, zeitgenössischer Relativismus, Rhetorik, klassischer Skepticismus, Soziologie der Erkenntnis.

Alban Bouvier, né en 1954, agrégé de philosophie, maître de conférences à Paris IVUniversité Paris-Sorbonne. Il a notamment publie L'Argumentation philosophique. Étude de sociologie cognitive (Paris, Presses universitaires de France, 1995) et son prochain ouvrage s'intitulera Philosophie des sciences sociales. Un point de vue argumentativiste en sciences sociales (Paris, Presses universitaires de France, à paraître).

Adresse : Paris IV-Université Paris-Sorbonne, GEMAS (CNRS), 54 bd Raspail, F-75006 Paris.

Courrier électronique : lecuyen@msh-paris.fr 
En écrivant L'Histoire du scepticisme d'Érasme à Descartes, devenu dans la traduction française, l'Histoire du scepticisme d'Érasme à Spinoza ${ }^{1}$, Richard H. Popkin a écrit un chapitre important de l'histoire des idées; son travail a été couramment interprété dans le cadre d'une histoire de la pensée scientifique et épistémologique et dans celui d'une histoire de la pensée religieuse, plus rarement dans celui d'une histoire de la pensée politique $^{2}$. Mon propos, dans cette brève contribution, est d'abord de suggérer l'intérêt que cet ouvrage peut représenter du point de vue d'une sociologie de la connaissance qui ferait de l'analyse des processus persuasifs et argumentatifs l'objet essentiel de sa démarche (I). J'essaierai de montrer ensuite comment on peut également reprendre et prolonger Popkin sur le problème particulier qui retient son attention, lequel relève plus spécifiquement d'une histoire des croyances (et des critères de validité de l'adhésion) puisqu'il s'agit de la compréhension du scepticisme au $\mathrm{xvII}^{\mathrm{e}}$ siècle (II). Enfin, je suggérerai la possibilité de prolonger «sociologiquement » Popkin du point de vue même d'une sorte d'archéologie du scepticisme contemporain (III).

\section{I. - HISTOIRE « PROBLÉMATOLOGIQUE 》 DES IDÉES, SOCIOLOGIE DE LA CONNAISSANCE ET « SCEPTICISME MODÉRÉ 》}

Dans le chapitre consacré à l'histoire des idées dans La Dynamique de la science, Larry Laudan fait de l'ouvrage de Popkin, en même temps que des Études sur le rôle de la pensée médiévale d'Étienne Gilson, des exemples vivants de ce que devrait être une bonne histoire des idées. Il écrit en effet :

« La tendance à ignorer les problèmes qui ont motivé l'élaboration des grands systèmes intellectuels du passé est un défaut persistant [...] dans la plupart des recherches sur l'histoire des idées [...] En effet, les systèmes de pensée ne sont pas seulement des liens logiques entre des propositions. Ils sont en plus des tentatives visant à résoudre ce qui est perçu comme étant des problèmes importants [...] Pendant plusieurs centaines d'années, les historiens des idées ont écrit sur la philosophie cartésienne [...] Et pourtant ce n'est que depuis peu que des chercheurs comme Gilson et Popkin ont commencé à éclairer les problèmes auxquels Descartes était confronte ${ }^{3}$.»

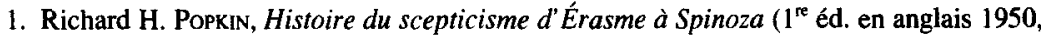
1964, 1979), trad. Christine Hivet, présentation Catherine LaRrère, Paris, Presses universitaires de France (Léviathan), 1995 (cité par la suite comme $H S$ ).

2. Cf. Catherine LarRére, présentation, in $H S$, et ID., L'Invention de l'économie au xvilf siecle, Paris, Presses universitaires de France, 1992, p. 20-21.

3. Larry Laudan, La Dynamique de la science (1 $1^{\mathrm{re}}$ éd. 1977), Bruxelles, Mardaga, 1987, p. 181. Laudan renvoie en note aux ouvrages que je signale. 


\section{Révolutions scientifiques, révolutions philosophiques}

Il y a, en fait, manifestement plusieurs raisons à l'intérêt que Laudan porte à Popkin, car l'un et l'autre se rencontrent sur plus d'un point. Popkin est lié, en effet, à la tradition épistémologique et sociologique à laquelle appartient Laudan (Thomas Kuhn, Paul Feyerabend, Imre Lakatos ${ }^{4}$ ), comme en témoigne le fait que Popkin a dédié son livre à la mémoire de Lakatos, dont, par son rationalisme, Laudan est le continuateur, ou le fait que Popkin a participé à un volume collectif de philosophie et d'histoire des sciences dirigé par Lakatos et par Alan Musgrave ${ }^{5}$. Une des raisons importantes de l'intérêt de Laudan pour Popkin est ainsi, fort probablement, même s'il n'en dit explicitement rien, le fait que, dans le modèle du développement des sciences que ce dernier propose, et qui repose sur une discussion serrée du modèle de Lakatos comme de celui de Kuhn, Laudan accorde une importance beaucoup plus grande que ces derniers (mais à peu près aussi grande qu'Alexandre Koyré) aux bouleversements philosophiques qui accompagnent les révolutions scientifiques. De ce point de vue, l'ouvrage de Popkin apporte une contribution substantielle à la compréhension des soubassements philosophiques de la révolution scientifique des $\mathrm{XVI}^{e}$ et $\mathrm{XvII}$ siècles, notamment par son analyse du rôle de Descartes dans cette révolution mais aussi, et peut-être surtout, des « libertins érudits » (Gabriel Naudé, Guy Patin, Léonard Marandé, François de La Mothe Le Vayer, Pierre Gassendi, Samuel Sorbière, Isaac La Peyrère) et du courant dit du « scepticisme modéré » (Pierre Gassendi, Marin Mersenne).

Les idées comme arguments, les arguments comme réponses contextuelles. Une approche "problématologique " de l'histoire et de la sociologie

Mais la raison sur laquelle Laudan insiste est pourtant sensiblement différente même si elle n'est pas sans lien avec la précédente; il s'agit en effet surtout de montrer que la dynamique de la science, le processus historique de sa construction progressive, n'est compréhensible que si l'on fait apparaître qu'il s'agit toujours de répondre à des problèmes, problèmes empiriques, bien sûr, problèmes conceptuels aussi et surtout (d'où l'importance du substrat philosophique). L'idée sous sa forme élémentaire et le précepte qu'elle implique peuvent paraître en soi triviaux (et Laudan le reconnaît), ils ne le sont nullement si on les prend réellement au sérieux. Ils impliquent manifestement un dépassement aussi bien d'une histoire prenant pour seul

4. Comme Kuhn, comme Feyerabend, voire comme Lakatos, Laudan introduit dans une interrogation d'origine épistémologique des considérations de type sociologique (par exemple, la notion de tradition de recherche, pendant de la notion kuhnienne de paradigme).

5. R. H. POPKIN, « Scepticism, theology and the scientific revolution in the seventeenth century ", in Problems in the philosophy of science, dir. Imre Lakatos et Alan Musgrave, Amsterdam, North-Holland, 1968, p. 1-39. 
objet l'étude des sources et des influences, que d'une analyse des philosophies comme simples systèmes ou architectoniques de concepts (à la façon de Martial Gueroult). Laudan formule ainsi la question qui devrait être fondamentale, selon lui, pour tout historien des idées : «Pourquoi tel penseur a-t-il adhéré, à un certain moment, à telles croyances? Pourquoi tel système de pensée a-t-il été modifié à un certain moment et à certains endroits ${ }^{6}$ ? » Le préfacier de la traduction en français du livre de Laudan, Michel Meyer, insiste fort justement, à mon sens, sur cette idée en prônant une sorte d'histoire "problématologique " " visant à repérer toujours à quelle « question » répondait le penseur dont les idées sont examinées. Par là, on n'entend pas seulement les questions que l'auteur se posait mais les questions qui lui étaient en quelque sorte posées par le milieu dans lequel il se trouvait, auxquelles il cherchait à répondre et dont la nature n'est pas toujours évidente à la lecture de la seule œuvre de l'auteur ${ }^{8}$.

Meyer, qui est un des héritiers à Bruxelles du fondateur de la « nouvelle rhétorique », Chaïm Perelman, montre alors comment une telle façon d'envisager la recherche historique ouvre vers « une conception rhétorique du discours » : «L'articulation des concepts s'opère en fonction de problématiques, ce qui est bien ce que l'on entend aujourd'hui par rhétorique. Il s'agit de répondre à ces questions et de les ordonner au sein de conceptualisations qui en traitent et les rapportent les unes aux autres ${ }^{9}$. » Et il est vrai que la focalisation de la recherche sur les raisons de l'adhésion ${ }^{10}$ oriente dans cette direction " argumentativiste ". Catherine Larrère, dans sa présentation de la traduction française de l'ouvrage de Popkin, va également dans le même sens pour caractériser la démarche de celui-ci : « L'approche de Popkin [...] consiste à rechercher la stratégie argumentative qui est à

6. L. LAUDAN, op. cit. supra n. 3 , p. 184.

7. «L'approche "problématologique", si l'on peut dire, de Laudan », voir op. cit. supra n. 3, p. 14. On peut tirer largement parti des conceptions épistémologiques et rhétoriques de Meyer (cf. Michel MeYER, Découverte et justification dans les sciences, Paris, Klincksieck, 1979; ID., Questions de rhétorique. Langage, raison et séduction, Paris, Le Livre de poche, 1993) sans pour autant adhérer à la métaphysique qui les prolonge ou cherche à les fonder (cf. M. MEYer, De la problématologie, Bruxelles, Mardaga, 1986). Sur ce point, je me permets de renvoyer à Alban Bouvier, "Pragmatique, rhétorique et sociologie de l'argumentation », in Approches pragmatiques, approches juridiques de l'argumentation, dir. Jean-Pierre COMETTI et Pierre Livet, Paris/Montréal, Vrin, à paraître.

8. Cf. le second exemple que L. LAUDAN, in op. cit. supra n. 3, p. 182, prend après celui de Descartes (étudié par Popkin et Gilson): "Bien que [la] littérature soit immense, nous n'avons toujours aucune idée claire de l'ensemble des problèmes auxquels Mill était confronté. Pourquoi, par exemple, a-t-il consacré autant de temps à ressusciter les méthodes d'induction par énumération et par élimination? Quels étaient donc les problèmes spécifiques dans les sciences sociales que sa célèbre "méthode historique" était supposée résoudre? "

9. Ibid., préf., p. 15.

10. Voir L. LaUdan, op. cit. supra n. 3, p. 184. 
l'œuvre dans un texte, à considérer celui-ci comme une réponse délibérée à une question posée dans une situation donnée ${ }^{11}$."

$\mathrm{La}$ question est de savoir si une histoire des idées aussi contextualisée par rapport aux questions des interlocuteurs réels ou virtuels, si intrinsèquement dialogique, est bien éloignée d'une sociologie dès lors que l'on assigne à celle-ci, à la manière par exemple de Georg Simmel ou de Gabriel Tarde, l'étude des interactions entre les individus ou lorsque, comme Tarde encore ou comme Vilfredo Pareto, on fait de l'étude des processus et des procédures de persuasion par lequel les idées et les croyances se transmettent un des objets centraux d'une sociologie des croyances ${ }^{12}$. Au demeurant, la dimension sociologique de l'œuvre de Perelman est ellemême au moins virtuelle. Dans un livre intitulé L'Art de se persuader, qui s'autorise d'une certaine lecture de Simmel et que d'autres ont présenté comme revitalisant les problèmes de Pareto et de Tarde ${ }^{13}$, Raymond Boudon renouvelle effectivement, quant à lui, et du point de vue de la sociologie de la connaissance, l'étude des procédures de persuasion et d'autopersuasion à la source de la production, de la réception et de la diffusion d'un certain nombre d'idées fausses, fragiles ou douteuses. On comprend, comme on le verra plus loin, qu'il en vienne assez naturellement à rencontrer aussi l'œuvre de Popkin sur son chemin ${ }^{14}$.

\section{L'héritage épistémologique et sociologique du scepticisme modéré}

On remarquera en passant que la proximité entre Popkin, Laudan, Lakatos, Perelman, Boudon est plus profonde encore. Dans son étude, Popkin oppose le scepticisme modéré (des pyrrhoniens) aussi bien au dogmatisme (de Descartes ou d'Antoine Arnauld) qu'au scepticisme radical (des acadé-

11. C. LaRrère, présentation, in $H S$, p. 13.

12. On pourra en rester, provisoirement au moins, à la solution simmélienne : histoire et sociologie ont la même matière mais l'histoire envisage celle-ci sous la catégorie ou la forme du devenir (elle focalise son attention sur les transformations, les processus) alors que la sociologie l'envisage sous celle des interactions (elle focalise son attention sur les relations que les individus entretiennent entre eux). Cela revient à établir une simple différence de polarité entre deux disciplines voisines, l'histoire sociologique et la sociologie historique, en considérant que la bonne histoire est forcément peu ou prou sociologique et la bonne sociologie peu ou prou historique.

13. Voir Giovanni Busino, "La destinée de la sociologie parétienne en France », L'Année sociologique, vol. 41, 1991, p. 205-227.

14. On pourrait également trouver une communauté d'inspiration entre ces recherches et la conception générale de la sociologie qui se dégage de certains travaux de Jean Baechler, comme recherche des problèmes que les sociétés ont à résoudre et des solutions qu'elles leur trouvent, voir Jean BAECHLER, La Solution indienne. Essai sur l'origine du problème des castes, Paris, Presses universitaires de France, 1988. Mais on a alors à faire à des problèmes qui dépassent de loin non seulement la seule histoire des idées mais aussi celle des microinteractions puisqu'il s'agit de situations où ceux qui posent les questions comme ceux qui $\mathrm{y}$ répondent sont anonymes; l'analyse ne peut être menée qu'à un niveau macrosociologique où l'étude des procédures d'argumentation et de persuasion n'a plus sa place. 
Il ne s'agit toutefois pas d'une histoire dont l'auteur s'abstrairait tout à fait puisque Popkin s'inscrit lui-même dans la continuité du scepticisme modéré dont il suit plus spécialement le développement; il voit en lui, en effet, le terreau même de l'esprit scientifique dont son étude prétend évidemment relever. Il suggère ainsi que le scepticisme modéré est à l'origine d'une tradition qui passe, selon lui, par Hume puis Stuart Mill, c'est-à-dire par la tradition empiriste. Popkin ne peut bien sûr connaître un élément de l'histoire de la sociologie, méconnu même des sociologues, à savoir que Pareto a construit une sociologie ou une anthropologie sociologique des croyances qui s'inscrit précisément dans la continuité expresse de Mill. Quoi qu'il en soit, on peut dire, selon moi, que tous ces auteurs, auxquels on pourrait ajouter Popper ou Hans Albert (dont nous reparlerons plus loin), se rattachent à ce scepticisme modéré, distant aussi bien du scepticisme radical (et quasi nihiliste) que des entreprises fondationnelles ( $" \mathrm{dog}$ matiques »); avec d'importantes nuances, bien entendu, depuis ce qu'il convient plutôt d'appeler un rationalisme modéré ou un rationalisme critique (Popper, Albert, Boudon) jusqu'au scepticisme modéré typique (Perelman probablement), opposant à la quête d'une inaccessible rationalité ultime la recherche modeste mais raisonnable d'arguments simplement vraisemblables ${ }^{15}$.

II. - HISTOIRE DES CROYANCES, SOCIOLOGIE DES CROYANCES ET ARGUMENTATION

L'EXEMPLE DU SCEPTICISME AU XVII ${ }^{e}$ SIÈCLE

Lorsque Laudan fait l'éloge de la manière de Popkin, il ne vise pas du tout, en tant que tel, le fait qu'il s'agit d'une histoire du scepticisme: la conception «problématologique » ou " argumentativiste » de l'histoire des idées ou de la sociologie vaudrait aussi bien pour n'importe quel autre objet (par exemple une histoire de l'induction ${ }^{16}$ ). Mais, en s'intéressant au scepticisme, Popkin aborde un problème d'histoire des idées d'un autre point de vue; ou, plus exactement, un problème d'histoire des croyances ou mieux encore, d'histoire des « attitudes de croyance » (et donc, symétriquement, non pas tant ou seulement de sociologie de la connaissance, mais de sociologie des croyances ou des attitudes de croyance) : il ne s'agit pas en effet de suivre l'évolution d'un concept ou d'une doctrine mais celle du degré de foi que des individus influents ou des groupes ont accordé ou

15. Voir $H S$, p. 49 , où le scepticisme modéré est identifié à « la quête du raisonnable ".

16. Cf. l'exemple de Mill, op. cit. supra n. 8. 
étaient prêts à accorder aux sciences, à la religion ou à la raison ${ }^{17}$. C'est là un objet qui est beaucoup plus rarement pris comme objet d'étude, au profit de la seule histoire des concepts. En s'attachant au niveau «doxastique » ou «épistémique " des croyances (plutôt qu'à leur niveau conceptuel ou représentationnel ${ }^{18}$ ), Popkin rencontre forcément, en outre, la question des critères de l'adhésion. Dès lors on a à faire, en quelque sorte, à une histoire (ou à une sociologie) argumentativiste des procédures argumentatives d'adhésion elles-mêmes, en tant qu'elles sont considérées ou non comme valides (par exemple, l'évidence rationnelle est-elle un garant de la validité des croyances ${ }^{19}$ ?). C'est là un objet d'investigation encore plus rare, aussi bien en histoire des idées qu'en sociologie de la connaissance ${ }^{20}$. D'où le caractère d'autant plus précieux du livre de Popkin.

\section{Dialogique intemporelle et sociologie formelle}

Voyons donc comment Popkin analyse globalement l'histoire du scepticisme au xviI siècle ou la trame générale de l'enchaînement de problèmes et de réponses successifs engendrés par la Réforme. De façon générale, Popkin caractérise comme crise sceptique l'ensemble des problèmes nés de la Réforme concernant les critères de l'adhésion. Le scepticisme était, en quelque sorte, une réponse spontanée au problème posé par l'apparition du dogmatisme calviniste, lequel introduisait, à la suite de Luther, un nouveau critère de la vérité, non seulement contradictoire avec celui du catholicisme mais conduisant en outre lui-même à la multiplication de ce qui pouvait prétendre être vrai, chaque conscience constituant en réalité, en l'absence d'un magistère, son propre critère. Mais ce scepticisme sans réel garde-fou avait des conséquences sur la foi en même temps que sur les sciences: il posait donc problème à son tour; par rapport à lui, le scepticisme modéré ou "constructif " s'offre alors comme réponse, mais cette réponse est jugée insuffisante et en appelle d'autres plus consistantes, comme celle de

17. Il ne s'agit pas tant d'étudier, par exemple, l'évolution de la notion de Dieu (d'un Dieu bon ou vengeur, par exemple, à un Dieu essentiellement architecte) que l'évolution du degré de croyance en Dieu (de la certitude de son existence au doute sur celle-ci, voire à la certitude de sa non-existence), même si les deux peuvent être liés. Exemplaire d'une articulation de ces deux niveaux pour une période un peu postérieure à celle qu'étudie Popkin est Bernard GroethuYsen, Origines de l'esprit bourgeois en France, T. 1: L'Église et la bourgeoisie

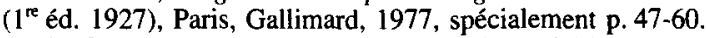

18. Ce niveau conceptuel ou catégorial est, évidemment, tout à fait digne d'intérêt aussi. J'ai essayé de montrer comment on peut également l'aborder d'un point de vue sociologique dans «Les formes philosophiques du point de vue d'une sociologie interactionniste », Kairos, 8, 1996, p. 11-33.

19. Voir aussi, in $H S$, p. 35 , cet autre exemple : «Luther définissait un nouveau critère de la liberté du savoir religieux : est vrai ce que la conscience est obligée de croire en lisant l'Écriture sainte. 》

20. Voir cependant Geoffrey E. R. Lloyd, Magie, raison et expérience. Origines et développement de la science grecque, Paris, Flammarion, 1990, chap. ॥, « Dialectique et démonstration ». 
Descartes. Pour résoudre le problème sceptique, Descartes choisit alors de pousser le scepticisme jusqu'à son terme, plus loin donc qu'il n'avait jamais été poussé (hypothèse du dieu trompeur, argument du malin génie) et de le renverser (grâce au cogito: «il n'y a donc point de doute que je suis, s'il me trompe »). Mais en échouant le plus souvent à convaincre de la validité de sa solution, Descartes aurait finalement rendu le problème plus aigu encore et ainsi renforcé la crise sceptique et préparé le scepticisme antireligieux.

C'est là une première manière de lire l'analyse problématologique de Popkin et qui caractérise incontestablement sa nervure centrale. En effet, le scepticisme antique offre un réservoir de stratégies argumentatives ${ }^{21}$ en quelque sorte intemporelles ${ }^{22}$, permettant de renforcer le problème sceptique ou, au contraire, de le modérer. On peut mettre en évidence, par exemple, la faiblesse des positions dogmatiques en montrant comment celles-ci reposent toujours soit sur une régression à l'infini soit sur un cercle vicieux ${ }^{23}$. La philosophie «dogmatique » depuis Platon, même si Popkin n'en dit rien, possédait aussi, du reste, un stock d'arguments permettant de « fonder » la vérité (par exemple, en bloquant la régression à l'infini par le recours à l'intuition d'une évidence inébranlable). Scepticismes variés (Montaigne, Mersenne) et dogmatismes divers (Calvin, Descartes) peuvent être ainsi mis en scène comme réponses aux problèmes respectifs qu'ils posent les uns et les autres, qu'ils posent les uns aux autres. Il $\mathrm{y}$ a là une sorte de dialogique intemporelle où sceptique et dogmatique se questionnent et se répondent dans une sorte de société philosophique purement idéelle. On aurait bien là encore un objet pour une sociologie, si l'on veut, mais en un sens proche de la sociologie formelle (ou « formale ») de Georg Simmel ${ }^{24}$.

\section{Sociologie matérielle et ethnologie des problèmes philosophiques}

Pourtant, Popkin va plus loin : il montre comment les arguments s'acclimatent en quelque sorte au contexte. Prenons ainsi l'exemple de l'argument du malin génie chez Descartes. Pour mieux percevoir son apport, voyons d'abord ce qui ne dépend pas du contexte dans cet argument (si ce n'est celui, très général ou très formel, de la réponse qu'est toujours, en quelque

21. Cf. notamment les Hypotyposes pyrrhoniennes de Sextus Empiricus, in Les Sceptiques grecs, textes choisis et trad. par Jean-Paul Dumonr, Paris, Presses universitaires de France, 1966.

22. Cela n'exclut évidemment pas qu'il y ait une histoire de la découverte de ces stratégies intemporellement valides.

23. Voir $H S$, p. 184.

24. Georg SimmeL, Questions fondamentales de la sociologie, chap. III, «La sociabilité. Exemple de sociologie pure ou formale », in Sociologie et épistémologie, textes de G. SimmeL, trad. par Julien FreUND, Paris, Presses universitaires de France, 1981, p. 121-136. 
sorte, le dogmatisme à un défi sceptique) : il ne suffit pas d'ébranler la croyance aux sens ou en la raison par des arguments rationnels mais il faut encore équilibrer la tendance, difficilement répressible, à refuser malgré soi son adhésion à des croyances familières dont on vient pourtant de montrer qu'elles sont douteuses. Le moyen de le faire est de chercher à se persuader que ce que l'on considérait auparavant comme vrai, ce que l'on devrait considérer maintenant comme douteux est en réalité radicalement faux. Il s'agit, en quelque sorte, de tordre le bâton dans l'autre sens et ainsi de vaincre, par une sorte d' "exercice », les résistances de l'habitude ${ }^{25}$. Quand la croyance est trop solidement fixée, il faut utiliser des stratagèmes qui vont bien au-delà des simples arguments logiques.

Mais différents contenus auraient probablement pu être donnés à ce stratagème visant les résistances des croyances familières aux meilleurs arguments. Il n'était pas nécessaire qu'il prenne la forme spécifique de l'hypothèse d'un malin génie. Celle-ci est en revanche explicable par le contexte historique particulier. Descartes ne répond pas, en utilisant l'hypothèse du malin génie, au seul problème en quelque sorte intemporel du fondement de la connaissance (réponse également quasi intemporelle au scepticisme, dans la dialogique d'une communauté idéelle) mais aussi à la forme historique que celui-ci prend à son époque. Or, le contexte de cette procédure rhétorique, dans la forme particulière qu'elle prend, c'est ce que l'on appelle l'affaire Grandier.

Il est rarissime qu'on fasse référence à cette affaire lorsqu'on essaie d'expliquer l'hypothèse du malin génie. Le procès de Loudun est un événement bien connu, notamment grâce au livre d'Aldous Huxley, que cite Pop$\mathrm{kin}^{26}$, mais c'est seulement chez Popkin, à ma connaissance, qu'on trouve énoncé le rapprochement avec l'hypothèse cartésienne du malin génie. Popkin rappelle en effet que « les années 1630 furent [...] marquées par le procès qui eut lieu à Loudun d'un prêtre du nom de Grandier qui était accusé d'avoir infesté un couvent de démons ». Popkin ${ }^{27}$ ajoute :

«L'affaire et les preuves qui furent présentées en 1634 lors du procès suscitèrent une vague de curiosité quant aux démons et aux preuves à l'appui desquelles de telles affaires pouvaient être jugées [...] Peut-être les implications de la fiabilité des preuves montrèrent-elles à Descartes que, s'il se pouvait qu'un

25. Michel Foucault oppose ainsi «trame démonstrative » et «trame ascétique », « système et exercice $»$ dans le cadre de sa réponse à la célèbre critique dont il a été l'objet de la part de Jacques Derrida quant au sens philosophique et historique à donner à la IV ${ }^{\mathbf{j}}$ Méditation; cf. Michel Foucault, Histoire de la folie à l'áge classique, Paris, Gallimard, 1972, appendice II, p. 594. Sur la pertinence de la distinction foucaldienne, cf. Jean-Marie BEYSSADE, " "Mais quoi? ce sont des fous. "Sur un passage controversé de la "Première Méditation" ", Revue de métaphysique et de morale, 78, 1973, p. 273-294.

26. Aldous HuxuEY, The Devils of Loudun, New York, 1952.

27. Voir $H S$, p. 237. 
pouvoir démoniaque existât en ce monde en dehors de l'affaire Grandier, il y avait tout lieu de se tourner vers le scepticisme. Il suffisait de considérer le niveau le plus général du raisonnement humain au lieu de la situation particulière des pensionnaires du couvent de Loudun pour être confronté à une possibilité stupéfiante : que nous en fussions conscients ou non, nous étions peutêtre tous victimes de démons; de surcroit, nous étions peut-être incapables de nous en apercevoir parce que les puissances démoniaques pouvaient exercer une illusion systématique."

Cette analyse relève entièrement, dans son principe, d'une sociologie ou d'une ethnologie de la croyance en un sens beaucoup plus habituel (ou matériel au sens de Simmel) du terme : Descartes donne un contenu à son argument sceptique en empruntant sa matière à un problème posé par le contexte de son époque quasiment dans les termes mêmes où il a été posé. On ajoutera que l'expression particulière que Descartes donne à son argument et qui a dû le rendre particulièrement parlant pour son époque le rend aussi particulièrement étrange pour la nôtre. D'un côté, en effet, on s'aperçoit que quand l'hypothèse d'un malin génie est discutée par les auteurs des objections, ce n'est jamais parce que l'idée d'un malin génie apparaîtrait invraisemblable : l'existence de celui-ci paraît au contraire faire partie de l'espace a priori des possibles; ce qu'on discute (Gassendi, Pierre Bourdin), c'est plutôt de la légitimité de considérer comme faux ce qui est simplement douteux. Mais, de l'autre, lorsqu'on a ou qu'on a eu pour charge d'enseigner à de jeunes esprits ce moment de la démarche cartésienne avec pour objectif, comme c'est l'esprit de l'enseignement de la philosophie en terminale en France, de faire revivre le doute cartésien, on a probablement été confronté au fait que cette hypothèse, plus encore que celle du dieu trompeur, paraît d'emblée invraisemblable (ce qui rend le procédé, en l'occurrence, assez inefficace).

La manière popkinienne de faire de l'histoire (sociologique) des idées (ou des croyances) est évidemment très différente de celle de Gueroult, comme on l'a déjà noté, puisque celui-ci n'étudie que la structure logique ou l'architectonique des systèmes philosophiques qu'il considère. Mais aussi de celle de Henri Gouhier qui, quoiqu'il ait été un des historiens de la philosophie les plus sensibles au problème de la communication de la philosophie, quoiqu'il ait même été un des premiers à intégrer la « nouvelle rhétorique » de Perelman à ses analyses, quoiqu'il soit aussi, en un sens, un disciple de Gilson, n'a probablement jamais fait de l'insertion dans les problèmes du contexte une règle de la méthode historique aussi nettement que celui-ci ou Popkin ${ }^{28}$.

28. Il serait injuste de ne pas rappeler que Gouhier a su éviter des écueils auxquels, par son « contextualisme", Gilson était plus naturellement porté : à savoir, interpréter les variations d'un auteur (par exemple, Descartes sur la liberté) comme de simples adaptations opportunistes au contexte. 
Effets contre-intentionnels de la critique du scepticisme : transgression des normes logiques ou transgression des normes sociales de la communication?

Il y a pourtant un point faible dans l'analyse de Popkin du cas Descartes, pour en rester au même exemple, mais qui est très instructif du point de vue d'une sociologie « argumentativiste » des croyances. Cette faiblesse apparaît beaucoup mieux maintenant, c'est-à-dire depuis les progrès qu'a faits l'historiographie cartésienne. Popkin réserve en effet deux chapitres à Descartes : dans l'un, il décrit la stratégie par laquelle Descartes a prétendu combattre et vaincre le scepticisme (le doute hyperbolique, le cogito et les preuves de l'existence de Dieu), dans l'autre l'échec final de Descartes à convaincre. Or, Popkin pense manifestement que l'échec de Descartes à réfuter le scepticisme est un échec logique, autrement dit que les arguments de Descartes étaient intrinsèquement non valides et que les auteurs des objections l'ont bien vu (notamment les «sceptiques modérés », comme Gassendi, récusant le doute hyperbolique, ou Mersenne, dégageant avant Arnauld les cercles vicieux cartésiens). D'autres commentateurs, notamment Jean-Marie Beyssade, ont montré de façon convaincante que l'argumentation de Descartes n'était pas du tout fautive, entendons logiquement fautive $^{29}$. Ainsi, on est conduit à comprendre autrement le processus qui a engendré l'échec de l'argumentation cartésienne et qui fait qu'il a bien œuvré malgré lui et même, peut-on ajouter, malgré le caractère logiquement valide de son argumentation, en faveur du scepticisme. Il apparaît alors forcément que le problème est un problème non pas de validité mais d'efficacité de l'argumentation, non pas un problème logique de rectitude de la pensée mais un problème sociologique de communication.

Mais l'inefficacité en question ne vient pas du tout ici de ce que Descartes aurait introduit des arguments mal contextualisés (voir au contraire, l'opportunité « ethnologique » de l'hypothèse du malin génie). À ce point, Popkin ne nous est plus d'aucun secours pour comprendre l'effet contreintentionnel qu'il repérait dans l'argumentation cartésienne parce qu'il ne s'est pas intéressé à la question générale des procédures efficaces de l'argumentation, qu'il s'agisse ou non d'une argumentation sceptique et quel qu'en soit le contexte : il ne s'est pas intéressé aux contraintes les plus générales (quoique très ordinaires) de la communication réussie - à la différence tant de Pareto que de Perelman. Il ne suffit pas, en effet, de dire que Descartes a été mal compris malgré la contextualité des réponses; il faut expliquer pourquoi. L'explication la plus répandue jusqu'à très récemment était de dire que c'était en raison de la précipitation et de l'inat-

29. J.-M. Beyssade, La Philosophie première de Descartes, Paris, Flammarion, 1979. 
tention des lecteurs et, au premier chef, des objecteurs. Certains (rares) commentateurs faisaient au moins (discrètement) exception, comme Ferdinand Alquié dans son édition des Euvres philosophiques de Descartes, quant au partage des responsabilités entre auteur et lecteur ${ }^{30}$. Depuis, notamment, un colloque qui s'est récemment tenu à Blacksburg en Virginie et qui était consacré aux Objections ${ }^{31}$, poursuivi par un colloque à la Sorbonne ${ }^{32}$, on assiste à une réhabilitation des auteurs de ces Objections, et, à travers eux, peut-on penser, de tous les lecteurs qui ont eu du mal à comprendre Descartes et à l'égard desquels l'argumentation a donc été, au moins en partie, inefficace. Cette réhabilitation est conforme à un usage équilibré du principe de charité dans l'interprétation; si on le met en œuvre, on s'aperçoit ainsi que ce ne sont probablement pas toujours les auteurs des Objections qui comprennent mal Descartes, c'est aussi Descartes qui, à son tour, comprend parfois mal les leurs. Mais le même principe invite à penser aussi que si les uns et les autres se sont mal fait comprendre, au point, comme Descartes, d'engendrer des effets contraires à ses intentions, c'est peut-être qu'ils se sont parfois mal exprimés, qu'ils ont transgressé certaines normes élémentaires de la communication du type de celles dont $\mathbf{H}$.-Paul Grice a donné un premier essai de systématisation ${ }^{33}$ (et que Boudon retrouve par une voie propre ${ }^{34}$ ) mais que nous connaissons tous intuitivement parce qu'elles relèvent des règles de la rhétorique élémentaire, à vrai dire élémentaire à formuler mais bien difficile, dans les faits, à toujours respecter. Je ne me livrerai toutefois pas ici à cette analyse du respect et de la transgression des normes sociales de la communication en philosophie parce que je l'ai donnée ailleurs ${ }^{35}$.

30. René DesCartes, Euvres philosophiques, textes établis, présentés et annotés par Ferdinand Alquí, Paris, Garnier Frères, 3 t., 1963, 1967, 1973.

31. Descartes and his contemporaries. Objections and replies, éd. Margaret Grene et Roger ARIEw, Chicago, University of Chicago Press, 1994.

32. Descartes. Objecter et répondre, dir. Jean-Marie Beyssade et Jean-Luc Marion, Paris, Presses universitaires de France, 1994.

33. Cf. H.-Paul GRICE, «Logique et conversation », Communications, 30, 1979, p. 57-72.

34. Raymond Boudon, L'Art de se persuader, Paris, Fayard, 1990, notamment chap. viII.

35. « La cohérence de l'argumentation philosophique et les normes de la communication. Une perspective de sociologie cognitive », in Descartes et l'argumentation philosophique, dir. Frédéric Cossutta, Paris, Presses universitaires de France, 1996, p. 43-84, contribution qui prend son point de départ dans le livre de Popkin. Voir aussi A. Bouvier, L'Argumentation philosophique. Étude de sociologie cognitive, Paris, Presses universitaires de France, 1995, qui expose de façon systématique le soubassement sociologique de cette analyse et propose un modèle d'étude des procédures d'argumentation en prenant comme exemple l'argumentation de Descartes dans les Méditations. 


\section{III. - ARCHÉOLOGIE DE LA MODERNITÉ \\ DU SCEPTICISME DES XVI ${ }^{e}$ ET XVII ${ }^{e}$ SIÈCLES AU SCEPTICISME CONTEMPORAIN}

L'ouvrage de Popkin est intéressant du point de vue de la conception interactionniste et problématologique de l'histoire des idées ou des attitudes de croyances et des critères épistémologiques; il l'est également du point de vue de l'analyse de cet exemple particulier que constitue l'histoire du scepticisme des $\mathrm{XVI}^{\mathrm{c}}$ et $\mathrm{xvII}{ }^{\mathrm{c}}$ siècles. Mais il est encore intéressant d'une troisième façon, par l'interprétation des origines de la modernité qui finalement s'en dégage. C'est donc du point de vue d'une archéologie des idées, comme aurait dit Michel Foucault, ou d'une sociologie historique du processus de modernisation, pour prendre un vocabulaire plus wébérien ou plus neutre, qu'on peut encore lire l'ouvrage ${ }^{36}$. Dans cette perspective, une des originalités les plus flagrantes de Popkin, en dehors du lien établi entre scepticisme et fidéisme et, inversement, de la présentation du scepticisme irréligieux comme d'une sorte de déviation tardive, est de lier scepticisme et modernité, alors que Weber comme Foucault (respectivement à propos de Calvin et à propos de Descartes) liaient essentiellement la modernité au développement du rationalisme. Popkin, comme on l'a noté, ne se contente pas, en effet, d'étudier la réapparition du scepticisme aux xvI et $\mathrm{xvIl}^{\mathrm{e}}$ siècles, il suggère également qu'on pourrait en suivre l'histoire ultérieure jusqu'à nos jours, à travers Hume et Mill, jusqu'au positivisme et au pragmatisme contemporains ${ }^{37}$. C'est ainsi manifestement l'histoire du scepticisme « modéré » ou " constructif » qui lui semble liée à l'essor de l'esprit scientifique et moderne, beaucoup plus que celle du rationalisme, qu'on suive la construction de celui-ci, comme Weber, à travers les grandes reli-

36. La référence que j'ai plusieurs fois faite à Foucault ne doit toutefois pas conduire à une méprise. J'y renvoie essentiellement en tant qu'il a formulé le programme, parfaitement estimable, d'une archéologie des idées. Mais, là encore, je suis globalement d'accord avec Laudan, malgré son ton polémique et son jugement très unilatéral dans sa critique de Foucault. Voir, par ex., L. Laudan, op. cit. supra n. 3, p. 244, n. 12 : «Comprendre un texte classique, selon Foucault, ce n'est ni le relier à la biographie de l'auteur, ni en examiner les arguments [je souligne]. En réalité, l'historien étudie de tels textes pour découvrir ce qu'ils nous révèlent sur la conscience [...] d'une époque. " Je laisse de côté ce qui concerne la biographie des auteurs pour retenir ce qui est dit de la place de l'analyse des arguments. Comme le suggère Laudan, Foucault, je crois, n'analyse pas réellement ceux-ci ou plutôt il n'analyse les énoncés, éventuellement argumentatifs, qu'en tant qu'on y découvre la marque ou le signe ou la trace de l'épistémè à laquelle ils appartiennent, non pas du tout en tant que ces arguments contribuent à construire et à transformer les représentations et les croyances d'une communauté. Voir aussi, in ibid., préf., p. 14, dans le même sens, les remarques de Meyer.

37. Ibid., p. 179. 
gions (notamment le calvinisme ${ }^{38}$ ) ou, comme Foucault, à travers la philosophie (Descartes étant un représentant exemplaire du basculement rationaliste).

On pourrait poursuivre la même histoire en s'intéressant de plus près à la vague contemporaine de scepticisme radical. Au niveau de la « dialogique intemporelle », on retrouve en effet, dans les problèmes que ce scepticisme pose et les réponses qui lui sont opposées, une configuration fort comparable à celle des $\mathrm{XVI}^{\mathrm{e}}$ et $\mathrm{XVII}{ }^{\mathrm{e}}$ siècles. C'est là aussi le motif le plus explicite de la référence de Boudon à Popkin. On a vu plus haut que la sociologie de la connaissance de Boudon pouvait s'articuler aisément sur le type d'histoire sociologique et problématologique que développe Popkin et j'ai suggéré tout à l'heure comment on pouvait prolonger les interrogations de Popkin sur la réception de la critique cartésienne du scepticisme en se centrant sur les procédures argumentatives appelées par la situation de communication dans sa généralité. Mais lorsque Boudon renvoie à Popkin dans L'Art de se persuader (au cours d'une simple et rapide note), c'est à propos d'une question différente quoique également très « popkinienne » : expliquer pourquoi le scepticisme épistémologique s'est imposé avec une telle force dans la pensée contemporaine, à la suite de Kuhn mais aussi et plus encore de Feyerabend ${ }^{39}$, c'est-à-dire de ceux-là mêmes que les rationalistes critiques ou les «sceptiques modérés » que sont Lakatos et Laudan ont critiqués avec le plus de constance et de fermeté. Et à cette fin, Boudon analyse, en sociologue de la connaissance attentif aux a priori linguistiques de la communication, les procédures argumentatives par lesquelles Feyerabend a pu persuader et, du reste aussi, probablement s'autopersuader de la validité du scepticisme radical qu'il prône ${ }^{40}$.

38. Dans la mesure où Calvin aurait construit une théologie offrant, à travers le dogme de la prédestination, une solution à l'antinomie du libre arbitre et de la préordination divine, antinomie présente dans toutes les théodicées mais rarement résolue, voir Max WEBER, Économie et société, Paris, Plon, 1971, p. 535-541.

39. R. Boudon, in op. cit. supra, n. 34 , p. 281 , n. 5 , renvoie plus précisément au « moule » qu'a constitué le raisonnement de Montaigne dans l'histoire du scepticisme. Dans l'ouvrage suivant, Le Juste et le vrai, Paris, Fayard, 1995, R. Boudon s'est intéressé à la vague symétrique de scepticisme et de relativisme moral qui a envahi des régions entières de la recherche intellectuelle. Il rencontre, là encore, un des axes de l'interrogation de Popkin; celui-ci renvoie, en effet, à l'un de ses articles sur « The philosophical base of modern racism », in Philosophy and the civilizing arts. Essays presented to Herbert W. Schneider on his eightieth birthday, éd. Craig Walton et John P. Anton, Athens, OH, Ohio University Press, 1974, en lien avec le débat sur le préadamisme d'Isaac La Peyrère, voir $H S$, p. 288, n. 62. Voir aussi la présentation de C. LARrère, in $H S$, p. 14.

40. R. Boudon, op. cit. supra n. 34, notamment p. 346-353. Le discours de Feyerabend est en fait hybride : l'anarchisme et l'irrationalisme méthodologiques qu'il prône à grands cris avec un sens très dadaïste de la provocation, dirigé essentiellement contre Popper et les poppériens (perçus par lui comme dogmatiques), voilent un pluralisme et un rationalisme méthodologiques nettement plus modérés (et aussi plus intéressants). 
On pourrait prolonger ce type d'analyse en se livrant, symétriquement, à l'analyse des arguments par lesquels le «dogmatisme» contemporain essaie de répondre à la crise sceptique. Comme Popkin pour Descartes, on pourrait alors se livrer à un exercice de « sociologie formelle » et montrer comment les interlocuteurs puisent, avec quelques innovations, les mêmes arguments dans le stock disponible. Lorsqu'on signalait plus haut la façon dont, au XVII ${ }^{e}$ siècle, on établissait la faiblesse des positions dogmatiques en montrant comment celles-ci reposent toujours soit sur une régression à l'infini soit sur un cercle vicieux, on aurait pu ajouter qu'il aurait suffi d'envisager la possibilité de décider de s'arrêter arbitrairement à un moment ou à un autre dans la remontée vers les principes premiers pour qu'on retrouve ce que Schopenhauer et Albert à sa suite appellent le trilemme de Münchhausen, c'est-à-dire la triple aporie auquel est censé être confronté tout projet fondationnel. La reconnaissance de l'existence d'un tel trilemme n'ouvre évidemment pas nécessairement vers un scepticisme radical mais aussi vers le scepticisme modéré ou le rationalisme tempéré d'un Albert. Chez Albert, toutefois, à la différence de Lakatos, Laudan ou Boudon, le principal adversaire n'est pas tant, on le voit, le scepticisme radical que le dogmatisme des philosophies du fondement, en l'occurrence, dans le contexte qui est le sien, celui de Karl-Otto Apel ${ }^{41}$. Resterait encore, comme Popkin le faisait à travers l'analyse de l'affaire Grandier, à montrer comment la nature des arguments est acclimatée au contexte spécifique, de sorte qu'on a à faire à une interaction argumentative entre des acteurs bien concrets.

Alban Bouvier (février 1997).

41. Voir, par ex., Hans Albert, La Sociologie critique en question, Paris, Presses universitaires de France, 1987, chap. Iv. 